Resection of ankle, with amputation if necessary, was proposed to and accepted by the parents. An incision was made over the sinus and the spongy periosteum raised from the bone; the sinus was then seen as a rounded hole leading into the end of the tibia; cutting forceps rapidly enlarged the aperture, and a small central necrosis surrounded by a dense caseous material was found in the end of the bone and removed. Incision was then prolonged along the inner side of the foot. The ankle-joint was dislocated outwards, cleaned, and examined. The cartilage of the tibial articulation lay loose in the cavity of the joint like a thin piece of orange-peel, and the astragalus itself was bare. The synovial membrane was everywhere transformed into the spongy, pink, gelatinous material characteristic of a tubercular joint. With a Volkmann's spoon, and here and there with a scalpel and forceps, this diseased structure was carefully removed. Finding the joint so distinctly tubercular, I re-examined the tibial wound and found more caseous material present in the medullary canal. I took a longhandled Volkmann's spoon and scraped away as much of the medulla up to the tibial tubercle as I could remove. The inside of the bone was then plugged with strips of iodoform gauze, and the foot brought into a rectangular position and there fixed by means of an appropriate splint. The wound was dressed with iodoform and wood-wool. The result exceeded my expectations : the medullary canal filled up with rapidity, the wound into the ankle-joint healed in two months, and the child was discharged with a gum-and-chalk casing. She is at the present time in apparently good health. She limps slightly, but this is due to a shortening which exists, the left leg having grown slightly longer than the right. The right tibia is thicker than the left one, and, curiously enough, there is some movement in the right ankle-joint, such as obtains in fibrous ankylosis. She wears an iron on that leg, to limit if possible the tendency to knock-knee on that side-a condition obviously induced by the difference in the length of the legs.

This case is a teaching one as regards the tolerance of the medulla. One is so taught to regard it as being highly resentful of interference that it has hitherto been treated with marked respect. The cases, however, in which the m edulla has been scraped show that good results have been obtained; and there is no doubt that much conservative surgery will be the direct outcome of the knowledge now accumulating as to its physiological uses, pathological tendencies, and the amount of operative interference it will submit to.

\section{A CASE OF PURPURA RHEUMATICA.}

By G. Childos Macdonald, M.D.

THE following case has recently occurred in my practice.

J. B- - aged twenty-nine, a blacksmith, married. Personal history satisfactory; does not remember ever having been ill, excepting cold, \&c. Both father and mother alive and in good health; four sisters alive and healthy; one brother dead. The patient does not know the cause of his illness, but believes it to be due to some childhood complaint. He had not been feeling well for the last week, having suffered from headache and sharp shooting pains in the back and down the legs; he describes them as rheumatic in nature, so much so that his friends were a rraid he was going to have an attack of acute rheumatism. Accompanying these pains was a sensation of cold, amounting occasionally to actual shivers; during this period he was extremely thirsty. The bowels were constip ated, and sleep was restless and broken. No beer or spirits had been taken, excepting a small amount of brandy when the shivers came on; neither did he have any drugs or particular article of food. At 12.30 P.M. on March 4th he vas taken with violent pain and stiffness in both popliteal regions, which became so agonising that he was unable to remain longer at work, and returned home, where, feeling rery cold, he sat by the fire, the pain varying in amount, being sometimes better and sometimes worse, until 8 P.M. when he determined on going to bed; he had difficulty in setting upstairs, the legs feeling stiff and heavy. While sitting on the edge of the bed previously to removing his txousers, the whole of the pain went as if by magic. On looking at his legs, he was frightened to observe that they had become blood-red, and being naturally alarmed he immediately sought medical assistance. The patient is a welldeveloped man. Height $5 \mathrm{ft} .8 \mathrm{in}$; weight $10 \mathrm{st} .10 \mathrm{lb}$. Skin somewhat pallid, but feels soft and moist. Pulse 82, quick, full, and hard; temperature beneath tongue $101^{\circ}$; tongue moist and clean. On the back of both thighs, extending from the gluteal folds downwards ovar the calves of the legs to about the middle, running to the inner surface of the thighs to the middle line in front, and on the legs to the crista of the tibix, is a red rash, most intense over the popliteal spaces, where it is uniform and somewhat raised; the rash on the inner surface of the thighs is similar in character; towards the periphery and in other places it is almost petechial. The whole of the involved areas of skin feel to the hand hot and dry; firm pressure has no effect on the colour, and it is unaccompanied by itching. The digestive, circulatory, respiratory, and genitourinary systems are normal. Urine clear amber colour; reaction acid; specific gravity 1020 ; no albumen; no sugar. The patient was ordered a saline mixture, with three-minim doses of tincture of aconite in each dose, a ten-grain pill of colocynth with calomel at bedtime, and a milk diet.March 5th: Has much improved; bowels relieved three times. Pulse 72 ; temperature $98^{\circ} 4^{\circ}$. There is no further extension of rash, which has become duskier in hue; no pain. Ordered a mixture of dilute sulphuric acid, tincture of perchloride of iron, and sulphate of quinine three times a day.7th: Patient feels quite well. The eruption has entirely disappeared. Ordered to continue mixture for three days. Ordinary diet.

The points of interest in this case are the violence of the pain, its rheumatic nature, its sudden cessation, its complete symmetrical arrangement, and the rapid recovery, which is usually prolonged in these cases. Dr. Kinnicutt of New York has recorded similar cases in the Archives of Dermatology, vol. i., as has also Dr. Duhring in the Philadelphia Medical Times, 1873.

Duchess-st., Cavendish-sq., W.

\section{CA SE OF $\mathrm{RHINOLITH}$}

By E. Cresswell Baber.

N. B---, aged twelve, was admitted a patient at the Throat and Ear Dispensary on Dec. 16th, 1886. She had had an offensive discharge from the left nostril for six years, with bleeding. There was no known cause. Neither she nor her mother remembered anything being put into the nose; but when young she had a habit of swallowing cherrystones. There is epiphora on the left side when she is exposed to the wind. On examination, the left side of the nose and the left cheek were bulged out; the left nasal passage was impervious to air, the nostril on that side being contracted and its edges excoriated. Anterior rhinoscopy showed, on the right, considerable deflection of the septum towards that side; on the left, the anterior naris blocked by large soft granulations. Behind these, and completely hidden from sight, the probe revealed a hard body about an inch and a half distant from the tip of the nose. On Dec. 18th, with the assistance of my colleague Mr. Treves, the patient being under chloroform, this body was removed through the nostril with scoop and forceps. Afterwards no roughness could be detected with a probe, and the instrument passed easily through into the naso-pharynx. Subsequent examination showed that the left nasal cavity was much dilated, allowing the naso-pharynx to be plainly seen through it. The inferior turbinated bone was much wasted, and presented a deep concavity about its centre. By cleansing the nostril and touching some remaining granulations with solution of caustic, the discharge has now (February 28th) almost ceased, and has entirely lost its disagreeable odour. No diseased bone has been detected. The left cheek still appears larger than the other, and the malar bone more prominent on that side, the inequality being apparently produced by the long sojourn of the nasal calculus. The rhinolith was more or less filbert-shaped, and had, as usual, a rough and mammillated surface. When dry it measured approximately $18 \times 13 \times 8$ millimetres and weighed nine grains. On section it was found to consist of tightly-

Read before the Brighton and Sussex Medico-Chirurgical Society March 3rd, 1887. 